DOI: https://doi.org/10.46630/phm.12.2020.03

Nikola R. Bjelić ${ }^{1}$

Article de recherche

Université de Niš

УДК 821.512.161.09-94 Pamuk О.

Faculté de philosophie ${ }^{2}$

Reçu : le 1/3/2020

Département de langue et de littérature françaises

\title{
LES ÉCRIVAINS FRANÇAIS DANS ISTANBUL D'ORHAN PAMUK
}

Publié en 2003, le livre Istanbul. Souvenirs d'une ville de l'écrivain turc contemporain le plus important, Orhan Pamuk (1952), est à la fois une biographie de la ville et une autobiographie de l'écrivain, dans laquelle il parle de son enfance et de son adolescence dans cette ville déchirée à l'époque entre l'Est et l'Ouest. En écrivant Istanbul, par le prisme de la mélancolie, Pamuk a pour idée de le situer dans la tradition des grands livres de mémoires, comme par exemple Confessions de Jean-Jacques Rousseau.

Les écrivains français que son père a traduits en turc (Valéry, Sartre, Simone de Beauvoir) occupent une place importante dans son éducation sentimentale et sa formation littéraire. Une analyse particulière dans Istanbul est consacrée aux écrivains français qui ont voyagé en Orient et qui ont visité Constantinople, en laissant des écrits sur leur fascination pour cette ville (Nerval, Gautier, Flaubert).

Dans cet article nous tenterons de montrer à quel point les écrivains français ont inspiré et influencé l'écriture de Pamuk, et d'analyser la manière dont son récit parle des deux mondes opposés auxquels appartient sa patrie : l'Orient musulman mystique et l'Occident rationaliste moderne.

Mots-clés : Pamuk, écrivains français, mémoires, Orient, mélancolie.

Peut-être aussi que la ville où nous vivons, tout comme notre famille, nous l'aimons parce que nous n'avons pas d'autre solution! Mais il nous faut inventer les lieux et les raisons à venir de notre amour pour elle. Orhan Pamuk, Istanbul (2007 : 532)

\footnotetext{
${ }^{1}$ nikola.bjelic@filfak.ni.ac.rs

${ }^{2}$ Cet article est rédigé dans le cadre du projet scientifique Les langues, les littératures et les cultures romanes et slaves en contact et en divergence (no 81/1-17-8-01) soutenu par l'AUF (Agence universitaire de la Francophonie) et l'Ambassade de France en Serbie.
} 


\section{Introduction}

Istanbul, ou Constantinople ${ }^{3}$, capitale de l'Empire ottoman et de la République turque, et avant ça de l'Empire byzantin (ou de l'Empire romain d'Orient), a toujours attiré l'attention des pèlerins, des croisés, des guerriers, des voleurs, des voyageurs qui y voyaient un espoir possible de succès. Pendant des siècles, des gens du monde entier venaient s'émerveiller devant « la beauté cosmopolite » (PAMUK 2007 : 320) de ce lieu unique au monde qui relie et sépare deux empires, deux continents, deux mondes, une multitude de peuples et de religions, celui qui relie et sépare l'Est et l'Ouest. C'est une « ville frontière (entre l'Orient et l'Occident)» (MOUSSA 2009: 9).

Depuis le XIX ${ }^{\mathrm{e}}$ siècle, l'Orient est le point d'intérêt de la société occidentale, surtout des écrivains et des humanistes français, anglais et plus tard américains, ce qui peut s'expliquer par le fait que l'Orient était (ou est encore) le territoire de la colonisation pour ces pays. Dans l'introduction de son célèbre livre L'Orientalisme (1978), Edward W. Saïd écrit que l'Orient était « une invention de l'Europe »", car c'est le « lieu de fantaisie, plein d'êtres exotiques, de souvenirs et de paysages obsédants, d'expériences extraordinaires » (2005: 13). À cause de cela, Saïd définit l'Orient par rapport à ce lieu particulier qu'il occupe dans l'expérience de l'homme de l'Occident, comme «l'une des images de l'Autre qui s'impriment le plus profondément en lui » (SAÏD 2005 : 14). La culture européenne, à partir du $\mathrm{XIX}^{\mathrm{e}}$ siècle, doit sa force et son identité « en se démarquant d'un Orient qu'elle prenait comme une forme d'elle-même inférieure et refoulée » (SAÏD 2005 : 16).

La diversité a toujours été l'une des caractéristiques les plus importantes d'Istanbul. C'est pourquoi les grands écrivains choisissaient souvent cette ville comme destination incontournable de leurs voyages en Orient. Istanbul était également un sujet d'intérêt des grands écrivains français du XIX ${ }^{\mathrm{e}}$ siècle, si bien que l'image de l'Orient et d'Istanbul apparaît dans leurs écrits. C'est d'abord Chateaubriand qui voyage en Orient entre 1806 et 1807 et décrit ce déplacement dans son célèbre Itinéraire de Paris à Jérusalem (1811), inspirant le désir des autres grands auteurs de découvrir cette partie du monde et d'écrire leurs relations de voyage. Après lui, Lamartine séjourne en Orient entre 1832 et 1833 et écrit son Voyage en Orient (1835). Nerval y voyage en 1843 et publie Voyage en Orient en 1851. Flaubert est en Orient entre 1849 et 1851 et il écrit sur ce sujet dans ses nombreux récits de voyage et dans ses lettres à sa mère et à Louis Bouilhet. Enfin, Gautier entreprend son voyage en Grèce et en Turquie en 1852 et publie son Constantinople en 1853.

Chateaubriand est l'initiateur et le fondateur de ce genre littéraire du voyage en Orient. Il inspire aux autres écrivains français l'écriture de leurs

\footnotetext{
${ }^{3}$ Constantinople fut l'ancien nom d'Istanbul, de 330 à 1930.

${ }^{4}$ Le sous-titre du livre de Saïd est en effet L'Orient créé par l'Occident.
} 
relations de voyage. Bien que les thèmes de ces ouvrages soient pour tous les mêmes, leurs récits sont différents, parce que leurs buts sont différents : tandis que « le pèlerinage de Chateaubriand est un voyage dans l'histoire, celui de Gérard de Nerval [est] dans le mensonge de l'histoire » (BUTOR 1972 : 17). Un point commun les réunit, c'est le désir d'écrire : « Ils voyagent pour écrire, et voyagent en écrivant, mais c'est parce que pour eux le voyage est écriture » (BUTOR 1972 : 17).

Puisqu'il s'agit d'un " lieu commun dans la littérature de l'époque » (ĐURIĆ 2019 : 317), il est tout à fait compréhensible que ces écrivains ont largement déterminé la vision occidentale d'Istanbul et de l'Orient aux XIX ${ }^{\mathrm{e}}$ et $\mathrm{XX}^{\mathrm{e}}$ siècles. Cependant, bien qu'ils aient souvent été fascinés par cette ville et ses beautés, leurs lecteurs développeront une image stéréotypée et souvent fausse de l'Orient et d'Istanbul, qui perdure jusqu'à nos jours.

Il serait intéressant de voir quel rôle ont joué ces écrivains et leur vision de l'Orient dans la formation et l'éducation d'un écrivain « oriental », turc, comme en témoigne le livre Istanbul. Souvenirs d'une ville de l'auteur contemporain Orhan Pamuk, publié en 2003 et traduit en français en 2007, un an après la remise par l'Académie suédoise du prix Nobel de littérature à ce romancier.

\section{La tristesse d'une ville}

Le livre de Pamuk contient de nombreuses références aux écrivains et peintres français. Comme il voulait être peintre de son enfance à ses 23 ans, il n'est pas étonnant qu'il se tourne souvent vers la peinture de peintres européens, en particulier vers les impressionnistes et les modernistes français. Cependant, les écrivains français occupent une place centrale dans ce livre autobiographique. Cela peut s'expliquer par son origine. Orhan Pamuk est né et a grandi dans une riche famille bourgeoise de la classe moyenne supérieure. Sa tante a terminé ses études de piano à Paris, alors que son père était un passionné de Paris, où il séjournait souvent, surtout lors de querelles avec sa mère. Ces dernières ont provoqué ses fréquentes absences, qu'il a passées principalement à Paris, où il croisait souvent, dans les années cinquante, Jean-Paul Sartre dans les rues (PAMUK 2007 : 468) et où il traduisait Valéry dans son hôtel (PAMUK 2007 : 468 ; PAMUK 2014 : 27, 650). À cause de l'admiration de son père pour la France, la bibliothèque dont disposait le petit Orhan était remplie de livres en français, qu'il lisait avec ferveur dans son enfance et son adolescence et qui lui témoignaient de ce qu'était la grande littérature, ce qui le conduit plus tard à devenir écrivain.

Orhan Pamuk est né en 1952, trois décennies après l'effondrement de l'Empire ottoman et l'instauration de la République turque par Mustafa Kemal 
Atatürk, qui souhaitait la rapprocher de l'Occident, l'européaniser et en faire un État démocratique moderne. Au cours de ces trois premières décennies de la «modernisation par l'occidentalisation » (DUCLOS 2013), la Turquie a connu des changements fondamentaux, mais au moment de la naissance de Pamuk, elle était déjà fatiguée des aspirations à une européanisation sans alternative, car il y avait, comme d'ailleurs aujourd'hui, un grand nombre de ceux qui n'aimaient pas beaucoup ce genre de rupture avec la tradition et un tel rejet de l'identité nationale. C'est pourquoi, bien qu'elle fût une ville énorme, l'Istanbul de l'enfance de Pamuk était un lieu très sombre, fatigué et mélancolique, loin d'être une ville moderne.

Cette mélancolie, hüzün en turc, est une des principales caractéristiques de l'Istanbul du $\mathrm{XX}^{\mathrm{e}}$ siècle. En écrivant le livre sur cette cité, Pamuk essaie de parler " du hüzün de toute une ville », de " ce sentiment sans pareil qui réunit la ville et ses habitants » (PAMUK 2007 : 131). Pamuk explique à quel point l'Istanbul de son enfance était indissociable de ce sentiment de tristesse qu'il produisait, et qui était expliqué le plus souvent par la chute de l'Empire ottoman. Cependant, le sentiment de tristesse peut être interprété de manières différentes. Il peut être un sentiment négatif, car il est lié à la maladie et à la souffrance, mais il peut également être interprété comme un sentiment positif, « comme un terme digne de considération et de nature poétique » (PAMUK 2007 : 129). Cependant, en attribuant ce sentiment à la ville, en disant qu'il s'agit d'un « état d'esprit » (2007 : 128), Pamuk distingue ce qu'on appelle communément la mélancolie, qui est « l'état mental d'une seule personne » (2007 : 138), de ce qu'on appelle la tristesse au sens employé par Claude-Lévi Strauss dans le livre Tristes tropiques (v. STRAUSS 1955). En utilisant le terme tristesse, Pamuk ne parle pas de la souffrance d'un seul individu qui peut être considérée comme un état douloureux, mais il l'associe à une culture, à un milieu et à un sentiment. Celui-ci se manifeste à l'Istanbul de son enfance, qui est très proche du temps où il représentait la capitale de l'empire. Ses monuments et ses bâtiments historiques sont donc visibles de tous, témoignant d'une grandeur qui s'est effondrée et qui n'est plus présente, et là-bas les gens ordinaires vivent. Le hüzün est né de l'oubli qui recouvre cette grandeur, de la pauvreté qui règne dans ces lieux magnifiques, de la négligence que le temps nouveau montre envers cette grandeur. C'est exactement ce qui était tant aimé et beaucoup apprécié par certains écrivains et certains voyageurs occidentaux, qui ont visité Istanbul au XIX ${ }^{\mathrm{e}}$ siècle :

À ce stade, ce qui différencie le hüzün de la tristesse ${ }^{* 5}$ est très net. La tristesse* qu'évoque Claude Lévi-Strauss, dans son ouvrage sans égal, est le sentiment que toutes ces pauvres grandes villes des tropiques, avec leur désarroi et leurs masses humaines, font éprouver à un Occidental. Le terme ne renvoie donc

${ }^{5}$ Le mot tristesse est utilisé en français par Pamuk. 
pas à l'état d'esprit de ces villes et de ceux qui y vivent, mais bien plutôt à la souffrance d'un Occidental débarqué dans ces villes, souffrance au dernier degré humaine, mêlant sentiments de culpabilité, tentative de s'affranchir des préjugés et des clichés, et pitié vécue. Le hüzün, pour sa part, est une réaction que développe non pas une personne qui regarde de l'extérieur, mais que développent les Stambouliotes à partir de leur propre situation. (PAMUK $2007: 142-143$ )

Cette tristesse est le sentiment majeur du livre de Pamuk. Écrivant sur ce hüzün, cette tristesse, Pamuk dit qu'Istanbul le porte non pas comme quelque chose d'imposé mais « comme quelque chose de sciemment choisi » (2007: 144). Le désir balzacien de réussir, élément déterminant de la ville moderne, est loin de cette tristesse. Parlant de tristesse, Pamuk mentionne de nombreux écrivains français. Outre les écrivains-voyageurs, dont nous parlerons plus tard, Pamuk mentionne parmi les premiers Montaigne, qui, très éloigné du concept de tristesse malgré sa propre mélancolie, pensait que la tristesse pouvait être surmontée par 1'《 intelligence d'intellectuel » (2007 : 147). Istanbul, au contraire, vit le hüzün et les premiers écrivains turcs modernes s'en rendent compte, parce que le hüzün est un sentiment prédominant dans leurs œuvres.

Ce qui est paradoxal, c'est que les quatre écrivains modernes stambouliotes qui « ressentent de l'enthousiasme pour la culture occidentale et désirent être modernes » (PAMUK 2007 : 149) donnent leur image d'Istanbul, ville où ils vivent, en étant inspirés par les écrivains-voyageurs français qui y séjournaient au XIX ${ }^{\mathrm{e}}$ siècle. Ces « quatre écrivains solitaires du hüzün »le mémorialiste Abdülhak Şinasi Hisar, le poète Yahya Kemal, le romancier Ahmed Hamdi Tanpınar et le journaliste-écrivain Reşat Ekrem Koçu - sont les quatre grands précurseurs de Pamuk, qui « ont été à un moment de leur vie éblouis par l'éclat de la littérature européenne, et tout particulièrement par celui de la littérature et des arts français » (Verlaine, Mallarmé, Valéry, Gide), s'efforçant « d'être modernes ou bien occidentaux dans leurs œuvres » (2007 : 154). Ces quatre écrivains inspirent le livre de Pamuk sur Istanbul. Il mène « un dialogue permanent » (PAMUK 2007 : 149) avec leurs œuvres. Ce dialogue avec eux est une sorte de mise en abyme, car c'est un dialogue avec leur vision de la vision d'Istanbul des écrivains-voyageurs français :

Ces quatre écrivains du hüzün, tout en créative complexité, pris entre passé et présent, entre Orient et Occident - selon l'expression favorite des Occidentaux-, m'ont fait aussi sentir comment je pouvais établir une relation entre la ville où je vivais et sa culture, les livres que j'aimais et mon goût pour l'art moderne. (PAMUK 2007 : 154)

Le livre de Pamuk représente donc également son dialogue avec ces écrivains français qui ont écrit avec enthousiasme sur Istanbul. 


\section{Melling dans Istanbul}

Avant de parler des écrivains français, Pamuk mentionne encore un Occidental qui était très important pour sa vision d'Istanbul et pour son livre, surtout grâce à ses paysages du Bosphore, Antoine Ignace (en allemand Ignaz) Melling, dessinateur, graveur, « un parfait Européen : d'origine italienne, [...] un Allemand de sang français » (PAMUK 2007 : 90). Melling est né en 1763 et, à l'âge de 19 ans, il entreprend un voyage qui le conduit à Istanbul en 1784, où il vécut pendant 18 ans, donnant des cours, proche de la haute société. Il était ami et menait une correspondance avec Hatice Sultan, sœur du sultan Selim III, dont il était l'architecte officiel. Pamuk mentionne le fait intéressant qu'eux deux échangeaient des lettres en turc écrites « avec l'alphabet latin, cent trente ans avant la 'révolution de l'alphabet' imposée par Atatürk » (2007 : 92), qui a remplacé l'écriture arabe par l'alphabet latin en 1928. En quittant la Turquie, Melling arrive à Paris où il publie en 1819 le livre Voyage pittoresque de Constantinople et des rives du Bosphore. Une édition phototypique de ce livre contenant 48 grandes gravures d'Istanbul a été publiée en 1969 par le poète et éditeur Şevket Rado, oncle par alliance de Pamuk. Ainsi ce dernier, à la période de son grand intérêt pour la peinture, a fait la connaissance de ce livre remarquable. Lorsque Pamuk regardait ces gravures, qui se caractérisent par une précision et une " minutieuse fidélité à la réalité » (2007:94), « le sentiment qu'Istanbul est sans centre ni fin s'installe en [lui], à la manière d'un conte d'enfance » (2007 : 96). Il lui arrive la pensée que le Bosphore était autrefois un paradis et que « sa vie est faite de certains de ces souvenirs, paysages et lieux de ce paradis perdu » (PAMUK 2007 : 97) :

Comme Marguerite Yourcenar l'a fait naguère avec les gravures de Rome et Venise entreprises par Piranèse trente ans avant Melling, moi aussi, « loupe à la main ", je prends un immense plaisir à contempler les Stambouliotes en mouvement dans les paysages d'Istanbul dessinés par Melling. (PAMUK $2007: 99-100)$

Puisque Melling a passé la moitié de sa vie à Istanbul, sa vie et sa vision du monde s'y sont formées. Ses gravures ne sont pas dramatiques, elles ne proviennent pas du romantisme oriental ou des clichés issus des Mille et Une Nuits, elles ressemblent plutôt à des miniatures islamiques, puisqu'elles proviennent directement de l'âme de la ville :

Mais comme à cette époque les habitants d'Istanbul ne savaient pas dessiner leur ville et sa population, et qu'ils ne s'intéressaient aucunement à ça, ce travail talentueux d'un Occidental créateur de représentations dénuées de tout jugement préconçu a été reçu comme un travail d'étranger. Parce que Melling dessine la ville à la façon d'un Occidental sans préjugés, qui voit la ville comme un Stambouliote, son Istanbul est un lieu familier, avec sa mémoire, sa géographie 
et ses mosquées, et, en même temps, c'est un monde sans équivalent, unique et pour cela extraordinaire. (PAMUK 2007 : 103)

Grâce à cela, Melling est selon Pamuk « le peintre auquel un Stambouliote peut s'identifier le plus facilement » (2007 : 98).

\section{Nerval et Gautier dans Istanbul}

La principale préoccupation de Pamuk dans Istanbul ce sont les trois écrivains-voyageurs français du XIX ${ }^{\mathrm{e}}$ siècle : Gérard de Nerval, Théophile Gautier et Gustave Flaubert. Pamuk consacre à ces trois écrivains, parmi les 37 chapitres de son ouvrage, 3 chapitres centraux, bien qu'ils soient omniprésents dans son autobiographie, surtout Flaubert.

Dans le chapitre 23, intitulé « Nerval à Istanbul : les promenades dans Beyoğlu », Pamuk parle du Voyage en Orient de ce grand poète français, qu'il lit « en relation, si ce n'est, peut-être, à travers deux écrivains turcs du XX $\mathrm{X}^{\mathrm{e}}$ siècle qui s'en sont inspirés », le poète Yahya Kemal et le romancier Ahmet Hamdi Tanpinar (MOUSSA 2009 : 5).

En parlant de Nerval, Pamuk souligne qu'il a fait son voyage en Orient pour trouver du réconfort, de l'oubli à sa mélancolie et pour échapper aux crises nerveuses dont il était auparavant victime. Il était en Orient en 1843, « un demi-siècle après que Melling a peint ses tableaux » (PAMUK 2007 : 285), mais il a publié son livre en 1851. Son voyage « est une quête à laquelle se livre le poète » inspiré par les voyages des « deux pères fondateurs du Voyage en Orient, Chateaubriand et Lamartine », s'intéressant en premier lieu « à la modernité des villes, à leurs habitants, leurs coutumes » (JULLIARD BEAUDAN 2010 : 114).

Nerval séjourne à Istanbul durant le mois de ramadan. Pamuk s'intéresse aux promenades de Nerval dans Beyoğlu, le quartier dans lequel il a grandi et passé toute sa vie, à proximité de la célèbre place de Taksim à Istanbul. À l'époque de Nerval, une centaine d'années avant la naissance de Pamuk, c'était le quartier chrétien de Péra, où se trouvaient les ambassades des pays occidentaux. En se promenant dans ce quartier et en recherchant le vieil Orient des époques de Chateaubriand et Lamartine, Nerval « prend acte de l'européanisation de la capitale ottomane » (MOUSSA 2009 : 3), amorcée par l'arrivée du sultan Abdülmecit en 1839. Cent ans plus tôt, l'endroit où se trouve la place de Taksim n’existait pas, ce n'était que des « planes étendues ». Pamuk dit:

Mais une phrase de Nerval à propos de ces planes étendues où je devais passer plus tard toute mon existence et qui m'apparaîtraient toujours « couvertes d'immeubles très anciens » reste imprimée dans mon esprit : «C'est un plateau immense ombragé de sycomores et de pins ! »(PAMUK 2007 : 286) 
Le traducteur de Pamuk en français traduit ici la phrase de Nerval du turc sans chercher l'original français. En fait, la phrase de Nerval est la suivante : « Dans une vallée étroite, ombragée de pins et de mélèzes » (NERVAL 1884 : 63). Pamuk explique comment la description nervalienne a provoqué sa propre mélancolie, voire sa douleur, car il s'est rendu compte que ce quartier était complètement différent de celui de son enfance et que l'une des parties les plus animées d'Istanbul à l'époque de Nerval n'était qu' " une vallée étroite ». Cependant, en tant que jeune homme, il s'intéressait à cette vision d'Istanbul touristique « diffusée par Nerval et beaucoup d'observateurs occidentaux, qui, s'inspirant tous les uns des autres, l'ont rendu exotique et suave » (PAMUK 2007 : 288), et qui intéressait Yahya Kemal et Tanpınar : ces derniers, dans un processus d'européanisation, cherchaient à trouver l'identité turque nationale et moderne en s'inspirant des écritures occidentales sur leur ville.

La description d'Istanbul par Gautier concerne également la mélancolie, à laquelle se réfère Pamuk dans le chapitre 24 , intitulé «Le périple mélancolique de Gautier dans les faubourgs ». Il passa 70 jours à Istanbul neuf ans après son ami Nerval, en 1852, exactement cent ans avant la naissance de Pamuk. Il a également séjourné à Istanbul pendant le ramadan. Puisque Gautier était journaliste, ses textes, plus élaborés et plus précis que ceux de Nerval, ont d'abord été publiés sous forme de feuilletons, puis dans le livre Constantinople l'année suivante. Selon Pamuk, il s'agit du meilleur livre sur Istanbul écrit au $\mathrm{XIX}^{\mathrm{e}}$ siècle après le livre Constantinopoli de l'Italien Edmondo de Amicis.

Gautier s'intéresse principalement aux faubourgs d'Istanbul, ses quartiers pauvres, miséreux, désolés et non européanisés, que les touristes ne visitaient pas, ce qui attire Pamuk beaucoup chez lui. C'est pourquoi le livre de Gautier, en plus d'être un guide de voyage, est en même temps une sorte d' « anti-récit de voyage » (MOUSSA $2009: 6$ ). Il erre dans les ruines, entre les tours et les murs byzantins, se demandant comment il est possible qu' " une cité vivante » puisse se cacher « derrière ces remparts morts » (GAUTIER : 231 ; PAMUK 2007 : 298), sans croire « qu'il y ait nulle part au monde une promenade plus austèrement mélancolique que ce chemin qui circule pendant près d'une lieue entre un cimetière et des ruines » (GAUTIER : 229 ; PAMUK 2007 : 298). Et, encore une fois, Pamuk réaffirme que le hüzün est le sentiment fondamental qui règne sur sa ville natale depuis plus de cent-cinquante ans, sans savoir expliquer pourquoi il est tellement heureux quand il en trouve la confirmation chez les écrivains étrangers :

Ce que j'essaie de raconter, c'est ce qui découle de l'invention, de l'expression et de l'invocation de ce sentiment en tant que concept, et que tout cela a été pour la première fois écrit par des poètes français renommés (notamment par Gautier sous l'influence de son mélancolique ami Nerval). Pourquoi ce que pensent Gautier et les Occidentaux auxquels je l'identifie à propos de ma ville, de la vie 
d'Istanbul et de ses spécificités a toujours été pour moi, et pour la ville, un sujet d'une telle importance ? (PAMUK 2007 : 299-300)

Dans le cas d'Istanbul et de la Turquie, qui luttent pour l'européanisation depuis le $\mathrm{XIX}^{\mathrm{e}}$ siècle, ce qui leur importe, c'est l'opinion des Occidentaux, ce qui confirmerait en quelque sorte qu'ils sont eux aussi « comme les Occidentaux » (PAMUK 2007 : 302). Cependant, l'opinion des Occidentaux peut être complètement différente. Alors que Pierre Loti aimait la Turquie pour son côté oriental et exotique, André Gide n'appréciait pas du tout les Turcs (PAMUK 2007 : 302-303). Pamuk déclare qu'il était toujours important pour les Turcs ce que les autres pensent d'eux :

C'est peut-être pour cela que, quelquefois, je lis ce que les voyageurs ont écrit sur la ville non comme le rêve exotique d'un autre mais comme s'il s'agissait de mes propres souvenirs. De plus, j'ai toujours beaucoup de plaisir quand l'observateur occidental perçoit et relate quelque chose que j'ai remarqué, mais que je n'ai pas conscience d'avoir remarqué parce que personne n'en parle. Par exemple : le constat par Knut Hamsun du léger balancement du pont de Galata soutenu par des bateaux à l'époque de mon enfance, ou le fait que Hans Christian Andersen écrive que les cyprès des cimetières sont « ténébreux ». Regarder Istanbul avec les yeux d'un étranger est toujours un grand contentement et une habitude particulièrement nécessaire face au sentiment communautaire et au nationalisme régnants. Parfois le harem ou les vêtements et les coutumes décrits avec réalisme me paraissent si éloignés de ma propre vie que, même si je sais parfaitement qu'il ne s'agit pas d'un rêve, tout ce qui est raconté m'apparaît comme le passé de la ville d'un autre. L'occidentalisation m'a donné, à moi et à des millions de Stambouliotes, le plaisir de trouver notre propre passé « exotique ». [...]

[L]'aliénation dans mon regard sur la ville, je l'ai acquise à force de lire les livres de voyageurs occidentaux. (PAMUK 2007 : 310-311, mis en gras par N. B.)

\section{Flaubert et l'art poétique pamukien}

Le troisième grand écrivain français à qui Pamuk consacre tout un chapitre est Gustave Flaubert. C'est le chapitre 31, intitulé « Flaubert à Istanbul : l'Orient, l'Occident et la syphilis ». Dans son texte prononcé à l'occasion de sa cérémonie de réception du titre de doctor honoris causa à l'Université de Rouen le 17 mars 2009, Pamuk dit qu'à la différence de Nerval qui était à Istanbul pour oublier sa mélancolie et ses crises nerveuses, Flaubert y séjournait alors qu'il était « préoccupé de ses problèmes personnels, de son avenir, de ses soucis » (PAMUK 2009). Flaubert était à Istanbul sept ans après Nerval et deux ans avant Gautier, aux mois de novembre et décembre 1850, avec son 
ami Maxime du Camp. Contrairement à eux, il ne s'intéressait pas beaucoup à Istanbul, parce que ce «n'était pas l'Orient qu'il recherchait » (PAMUK 2007 : 355). Étant donné qu'il a contracté la syphilis lors de son voyage en Orient, plus précisément à Beyrouth, ses écrits sur Istanbul, disséminées dans ses lettres, témoignent de son inquiétude pour « son phallus, qui est à l'époque sa plus grande préoccupation » (PAMUK 2007 : 359). Dans sa célèbre lettre à Louis Bouilhet du 14 novembre 1850, dans les paragraphes que les éditeurs suppriment le plus souvent ${ }^{6}$, il parle de sept chancres sur son phallus qui se sont regroupés ensuite en un seul (PAMUK 2007 : 359 ; FLAUBERT 1974 : 94). Pourtant, il ne refuse pas à Istanbul, comme à Beyrouth avant cela, de se rendre dans un bordel où une fille de seize ou dix-sept ans lui a plu. Dans la lettre à Louis Bouilhet du 15 décembre, il décrit cette visite au bordel : quand cette fille lui demande de lui montrer son membre pour vérifier s'il n'est pas malade, il lui répond qu'elle l'a offensé et quitte le bordel (PAMUK 2007 : 360 ; FLAUBERT 1974 : 108).

Cependant, Flaubert est pour Pamuk le plus important de tous les écrivains ayant visité Istanbul. Dans le chapitre consacré au séjour de Flaubert à Istanbul, ainsi que dans le texte de réception du titre de doctor honoris causa, Pamuk expose son art poétique, qui est largement inspiré par Flaubert. Citant un extrait de la lettre de Flaubert adressée à sa mère le 15 décembre 1850, dans laquelle il refuse les contraintes, les emprises de la société, l'avenir, les institutions et même le souci de sa possible gloire littéraire, Pamuk dit qu'il lui « arrive parfois de songer à ces lignes, pensées et écrites cent deux années avant [s]a naissance dans les rues où [il] allai[t] passer toute [s]a vie, et qui allaient représenter le fondement de la pensée de la littérature moderniste » (2007 : 356). Dans ses lettres envoyées d'Orient, Flaubert formule souvent ce qui lui est important dans l'art et dans la littérature et « ses propres projets littéraires » (MOUSSA 2009 : 10), ce qui le place plus tard " en tête des auteurs vénérés par d'autres écrivains » (PAMUK 2009). Il est l'un des auteurs qui apporte le modernisme à la littérature turque ainsi qu'à toutes les autres littératures. Flaubert représente pour Pamuk « un saint autant qu'un ermite : le premier à vrai dire de ces saints ermites de la littérature moderne qui ont tourné le dos à la vie et au succès superficiel » (PAMUK 2009), un des rares écrivains auxquels beaucoup d'auteurs ont voulu s'identifier, s'identifier à la fois « à l'écrivain en colère, profondément irrité contre l'humanité, et à celui qui, nourrissant une telle tendresse pour ses semblables, les comprend mieux que quiconque » (PAMUK 2009). C'est pourquoi Pamuk dit, en paraphrasant Flaubert, que « Monsieur Flaubert, c'est moi !» (2009).

${ }^{6}$ Voir les notes à la page 11 (FLAUBERT 1974). 


\section{En guise de conclusion}

Pamuk mentionne dans son livre beaucoup d'écrivains français (Valéry, Sartre, Loti, Camus, Baudelaire, Hugo, Mallarmé, Gide, Vian, etc.). D'ailleurs, « le panthéon littéraire national est principalement composé des grands auteurs étrangers, en particulier français » (DUCLOS 2013). Parmi eux, ces trois grands écrivains et voyageurs français qui ont laissé leur témoignage écrit sur sa ville natale occupent une place majeure dans cette autobiographie, ainsi que dans sa formation littéraire.

Le regard des Occidentaux sur l'Orient est particulièrement important pour sa formation. Il s'intéresse tant à ces écrivains qu'il s'identifie parfois à eux, parce qu'il a dû « [s]e former en [s]e faisant influencer et en [s]e confrontant à eux ", mais " aussi parce que les voyageurs occidentaux [lui] ont appris plus de choses au sujet des anciens paysages d'Istanbul et de son quotidien que les écrivains stambouliotes qui ne prêtaient aucune attention à leur ville» (PAMUK 2007 : 356-357), concluant :

leurs imaginations, leurs interrogations, la volonté d'expansion de leurs États, ou encore la curiosité pour ce qui se trouvait au-delà de leurs frontières les ont poussés à se rendre à l'endroit qui s'appelle mon « chez-moi ». Ils ont consigné ce qu'ils y ont vu, et mon monde s'est infiltré dans leurs écrits et leurs images. (PAMUK 2007 : 358)

On peut dire aussi que leurs visions de son monde se sont infiltrées dans ses écrits. Pamuk dialogue incessamment dans son livre avec ces trois auteurs français, parce que leur opinion lui est importante «pour mieux comprendre sa ville natale » (MOUSSA 2009 : 12). Comme leur opinion, qui influençait la formation de Pamuk, le livre de ce dernier « nous oblige à relire ceux-ci différemment $\gg$ (MOUSSA 2009 : 12). En se référant à ces auteurs, Pamuk a montré que l'Istanbul du XIX ${ }^{\mathrm{e}}$ siècle était une ville beaucoup plus cosmopolite que l'Istanbul de son enfance (PAMUK 2007 : 308).

On pourrait conclure que c'est la littérature de Pamuk qui, grâce aussi au Prix Nobel qu'il a obtenu en 2006, «mondialise [...] l'espace littéraire turc » (DUCLOS 2013). Chez Pamuk, Istanbul « devient un véritable personnage littéraire » (MOUSSA 2009 : 13). Ainsi cette autobiographie de l'écrivain se transforme en une vraie biographie de cette ville. C'est une des qualités majeures de ce grand livre pamukien.

\section{Bibliographie}

BUTOR 1972 : BUTOR, Michel. «Le voyage et l'écriture». In : Romantisme. « Voyager doit être un travail sérieux ». No4, 1972:4-17. 
DUCLOS 2013 : DUCLOS, Elise. «'En lisant en écrivant' : Orhan Pamuk auteur de la littérature européenne ». In : Les Cahiers $d u$ CERACC. Paris : Université Paris 3. No 6, juillet 2013. <http://www.cahiers-ceracc.fr/ duclos.html> 10. 02. 2020.

ĐURIĆ 2019 : ĐURIĆ, Vladimir. « L'image de l'Orient dans les récits de voyage de Chateaubriand, Lamartine, Nerval, Gautier et Flaubert ». In : Facta Universitatis : Series linguistics and Literature. Réd. Nikola Bjelić et Ivan Jovanović. Niš : Univerzitet u Nišu. Vol. 17, nº. 2, 2019 : 309-318.

JUILLIARD BEAUDAN 2010 : JUILLIARD BEAUDAN, Colette. " Le voyage en Orient de Gérard de Nerval ». In : Les Cahiers de l'Orient. ${ }^{\circ}$ 97, 2010/1 : 113-121.

MOUSSA 2009 : MOUSSA, Sarga. «Orhan Pamuk lecteur des écrivains voyageurs français a Constantinople au XIX $\mathrm{X}^{\mathrm{e}}$ siècle ». https://halshs. archives-ouvertes.fr/halshs-00415857/document 10. 02. 2020.

PAMUK 2009 : PAMUK, Orhan. « Monsieur Flaubert, c'est moi ! ». Traduit par Gilles Authier. Rouen : Université de Rouen, 2009. <https://flaubert. univ-rouen.fr/etudes/pamuk_francais.php $>10.02 .2020$.

PAMUK 2014 : PAMUK, Orhan. D’autres couleurs. Traduit par Valérie GayAksoy. Paris : Gallimard, 2014. Epub.

SAIID 2005 : SAÏD, Edward. L'Orientalisme. Traduit par Catherine Malamoud. Paris : Seuil, 2005.

STRAUSS 1955 : STRAUSS, Claude-Lévy. Tristes tropiques. Paris : Plon, 1955.

\section{Sources}

GAUTIER 1853 : GAUTIER, Théophile. Constantinople. Paris : Michel Lévy frères, 1853.

FLAUBERT 1974 : FLAUBERT, Gustave. Correspondances. Vol. 2 (18501859). Paris : Club de l'Honnête Homme, 1974.

NERVAL 1884 : NERVAL, Gérard de. Voyage en Orient. Vol. 2. Paris : Calmann Lévy, 1884.

PAMUK 2007 : PAMUK, Orhan. Istanbul. Souvenirs d'une ville. Traduit par Savas Demirel, Valérie Gay-Aksoy et Jean-François Pérouse. Paris : Gallimard, 2007. Epub. 
Никола Р. Бјелић

\section{ФРАНЦУСКИ ПИСЦИ У ИСТАНБУЛУ ОРХАНА ПАМУКА}

Објављена 2003, књига Истанбул. Успомене и град најважнијег турског савременог писца, нобеловца Орхана Памука (1952), у исто време је и биографија града и аутобиографија писца, у којој он говори о сопственом детињству и одрастању у овом граду који је у то доба био располућен између Истока и Запада. Пишући Истанбул кроз призму меланхолије, Памук има идеју да ову књигу смести у традицију великих мемоарских књига, као што су биле, на пример, Русоове Исповести.

Француски писци, које је Памуков отац преводио на турски (Валери, Сартр, Симон де Бовоар), заузимају важно место у његовом сентименталном васпитању и књижевном формирању. Посебно место је у Истанбулу посвећено француским писцима XIX века који су путовали на Исток и који су забележили своје дивљење према овом граду (Нервал, Готје, Флобер).

У овом раду покушали смо да покажемо у којој су мери ти француски писци инспирисали Памука и утицали на његово књижевно стваралаштво, као и да анализирамо начин на који ово дело говори о два супротстављена света којима његова домовина припада, а то су мистични муслимански Оријент и рационалистички модерни Запад.

Кључне речи: Памук, француски писци, мемоари, Оријент, меланхолија 